\title{
FACTO RES AMBIENTALES Y GENÉTICOS RELACIONADOS CON EL INTERVALO ENTRE PARTOS EN LA RAZA ROMOSINUANO
}

\author{
ENVIROMENTAL AND GENETIC ASPECTS RELATED WITH CALVING \\ INTERVAL IN ROMOSINUANO BREED
}

\author{
Gustavo 0 ssa $\mathrm{S}^{1 *}$, Marco Suárez $\mathrm{T}^{2}$, Juan Pérez $\mathrm{G}^{1}$ \\ 1Programa Nacional de Recursos G enéticos y Biotecnología Animal, CORPO ICA. C.I. Turipaná. Montería, \\ Córdoba - Colombia. Universidad Agraria de La Habana². Facultad de Medicina Veterinaria, Departamento \\ de Producción Animal. La Habana, Cuba. *Correspondencia: sarazgossa@ yahoo.es
}

Recibido: Marzo 31 de 2006; Aceptado: Noviembre 30 de 2006

\section{RESUMEN}

0 bjetivo. Estudiar los factores ambientales y genéticos que influyeron sobre el intervalo entre partos de hembras romosinuano. Materiales y métodos. Se analizaron retrospectivamente 1.911 registros los intervalos entre partos de las vacas de la raza Romosinuano del Centro de Investigaciones Turipaná de CORPO ICA, Cereté, Córdoba, desde el año de 1980 hasta el año 2003. Para el estudio de los factores ambientales se utilizó el método de los cuadrados mínimos mediante el procedimiento PRO C GLM de SAS. Resultados. La media estimada para el intervalo entre partos fue de 433,60 \pm 3,69 días, con una desviación estándar de 139,18 días y un coeficiente de variación de $32,10 \%$. La heredabilidad estimada a través de los componentes de varianza del méto do MTDFREML fue de 0,05 $\pm 0,04$. Conclusión. El análisis de varianza reveló que el año, el mes de nacimiento y el número de partos, fueron causas estadísticamente significativas de variación del intervalo entre partos.

Palabras clave: Herencia, ambiente, intervalo entre partos, romosinuano.

\section{ABSTRACT}

0 bjective. To study the environmental and genetic factors that influence on the calving interval of romosinuano females bovines. Materials and methods. 1.911 data were analyzed retrospectively the interval calving of romosinuano females bovines from a herd at the Turipaná Experimental Station of CORPO ICA, C ereté, Colombia, from 1980 until 2003. For the study of the environmental factors the method of the minimum squares was used by means of the PRO C G LM of SAS procedure. Results. The estimated mean for the interval calving was of $433,60 \pm 3,69$ days, with a standard deviation of 139,18 days and a coefficient of variation of $32,10 \%$. The estimated heredity through the components of variance of the MTDFREM L method was of $0,05 \pm 0,04$. Conclusion. The variance analysis revealed that the year, the month of birth and the calving number were causes statistically significant of variation of the interval calving.

Key words: Heredity, environment, calving interval, romosinuano 


\section{INTRODUCCIÓN}

El intervalo entre partos constituye el carácter más importante en la evaluación de la eficiencia reproductiva individualmente en las vacas dentro de un hato, ya que la duración ideal es de 365 días, es decir un ternero/vaca/año. Al considerar que el intervalo entre partos esta formado por dos componentes (período de servicio más período de gestación), esto indica que una vaca deberá ser fecundada alrededor de los 85 días después del parto, si se tiene en cuenta que el perío do de gestación de las vacas es de 284 días aproximadamente. El corto intervalo entre partos es uno de los caracteres por medio del cual las razas nativas han mostrado su adaptación a las condiciones ecuatoriales, y por lo tanto se considera de importancia su estudio en la caracterización de las mismas.

La identificación correcta de los efectos de los factores ambientales y genéticos responsables de las variaciones en los intervalos entre partos, permite establecer planos adecuados en la creación, con el objetivo al mejoramiento de este carácter.

En la tabla 1 se presentan los valores medios encontrados para el intervalo entre partos en diferentes razas nativas en Colombia y en algunos países localizados también en zonas tropicales.

De otra parte, entre los factores el sexo y a mbientales que han sido relacionados como causa de variación en los intervalos entre partos se destacan los siguientes: año, mes de parto, orden del parto y edad.

Respecto a las diferencias en el intervalo entre partos relacionadas con el año y/o mes del parto son atribuidas, básicamente, a las fluctuaciones de alimentación que ocurren de año en año o entre meses en el mismo año.

Tabla 1. Intervalos entre partos de algunas razas en diferentes países

\begin{tabular}{llll}
\hline Raza & IEP (días) & País & Referencia \\
\hline Kouri & 456 & India & Bourzat et al. (1) \\
Caracú & 432 & Brasil & Dias et al. (2) \\
Nelore & 410 & Brasil & Padua et al. (3) \\
Caracú & 448 & Brasil & Pereira et al. (4) \\
Sanmartinero & 469 & Colombia & Martínez et al. (5) \\
Bo N & 486 & Colombia & Tobón et al. (6) \\
Nelore & 383 & Brasil & Bergmann et al. (7) \\
Gir & 496 & Brasil & Balieiro et al. (8) \\
Lucerna & 382 & Colombia & Durán y Manrique (9) \\
Sanmartinero & 517 & Colombia & Martínez (10) \\
Hartón del Valle & 375 & Colombia & Alvarez (11) \\
Costeño con Cuernos & 446 & Colombia & O ssa (12) \\
Velásquez & 420 & Colombia & Velásquez (13) \\
Sanmartinero & 499 & Colombia & Martínez y González (14) \\
Nelore & 432 & Brasil & Cavalcante et al. (15) \\
Sahiwal & 420 & India & Joshi et al. (16) \\
Ongole & 502 & India & Gaur et al. (17) \\
Brahman & 431 & Colombia & O ssa et al. (18) \\
Deoni & 447 & India & Singh et al. (19) \\
\hline
\end{tabular}

$\mathrm{IEP}=$ intervalo entre partos. 
Con respecto al efecto del año, Hernández et al. (20) no encontraron efecto con respecto al intervalo entre partos en la raza Romosinuano. En contraste, Pereira et al. (21); Días et al. (2); Gómez et al. (22) y Tobón et al. (6) lo reportaron en las razas Caracú, Sanmartinera y BON, respectivamente. En la raza $\mathrm{N}$ elore, también se ha encontrado dicho efecto $(3,7,15)$.

En cuanto al efecto del mes sobre el intervalo entre partos se ha evidenciado que en general, en los meses correspondientes a la época de invierno se encuentran los menores intervalos entre partos $(2,20,21)$. En la raza $N$ elore varios autores observaron efecto significativo del mes sobre el intervalo entre partos $(3,7,15)$.

Respecto a la edad de la vaca u orden de parto ha sido una causa de variación en los intervalos entre partos intensamente estudiada. Al respecto, los resultados presentan controversias. Hay una relativa uniformidad de que el primer intervalo entre partos es mayor que los demás $(4,10,21)$.

El efecto atribuido al sexo del ternero como causa de variación con respecto al intervalo entre partos ha sido explicado de diferentes maneras. El nivel nutricional durante y después de las gestaciones, duración de la gestación con efecto en la duración del período de servicio y en el aumento del número de servicios por concepción son tentativas de explicación de este efecto.

En la raza Caracú, se encontró que el efecto del sexo del ternero fue altamente significativo, ya que las vacas que parieron machos presentaron un intervalo mayor a las que parieron hembras (21). También en la raza Nelore se observó el mismo efecto (7).

El objetivo de este trabajo retrospectivo fue el de a nalizar los factores a mbientales y genéticos que influyeron sobre el intervalo entre partos de hembras romosinuano.

\section{MATERIALES Y MÉTODOS}

Tipo de estudio. Se analizaron retrospectivamente 1.911 registros de intervalo entre partos, de la raza Romosinuano, comprendidos entre el año de 1981 y 2003.
Área geográfica, clima, sitio de estudio y tipo de ganado. El trabajo se realizó en el Centro de investigación Turipaná, en el municipio de Cereté, Colombia. Este presenta topografía plana, con una altitud de $20 \mathrm{msnm}$. Temperatura promedio anual de $280 \mathrm{C}$, humedad relativa entre 79 y $84 \%$ y precipitación pluviométrica de $1120 \mathrm{~mm} / a$ ño. Los pastos predominantes son angletón (Dichatum aristtum) y Tanzania (Panicum sp). El sistema de monta es natural y es restringido a cuatro meses del año entre abril y julio, para que la estación de nacimientos coincida con el período seco del año, cuando la mortalidad de los terneros es menor.

El sistema de pastoreo durante la estación de monta es alterno y después se adopta el rotacional. Cada potrero tenía una extensión de cinco hectáreas. Las vacas y novillas fueron suplementadas en la época seca con ensilaje de maíz. Las novillas fueron colocadas con los toros cuando alcanzaron una edad de dos años; el sistema de apareamiento es circular cíclico y ningún toro con un grado de parentesco estrecho o de la misma familia de las hembras se aparearon entre sí. Los cuidados sanitarios y profilácticos de rutina fueron llevados a cabo.

Análisis de datos. Para el a nálisis de los factores ambientales se utilizaron análisis de varianza mediante modelos lineales utilizando el procedimiento PRO C GLM, contenido en el Statiscal Análisis System (23).

El modelo matemático para el análisis del intervalo entre partos, incluyó los efectos fijos de año y mes del parto, orden del parto y sexo del ternero.

$$
\begin{aligned}
& Y_{i \mathrm{ijk} m}=\text { intervalo entre partos. } \\
& i^{i j k l m}=\text { media general. } \\
& A_{i} \quad=\text { efecto del año del parto (1981, }
\end{aligned}
$$

El número de partos se agrupó en 4 clases: Clase $1=$ vacas de segundo parto, clase $2=$ vacas de 
tercer parto, clase $3=$ vacas de cuarto parto y clase $4=$ vacas de 5 y más partos.

Las heredabilidades fueron calculadas de los componentes de varianza obtenidos del modelo animal. El programa utilizado fue MTDFREM L (24).

\section{RESULTADOS Y DISCUSIÓN}

En la tabla 2 se observa el resultado del análisis de varianza de los intervalos entre partos, de acuerdo con el modelo planteado.

La media del intervalo entre partos ocurridos de 1981 a 2003, fue de 433,60 $\pm 139,18$ días, y un coeficiente de variación de $32,10 \%$. Este resultado es similar a los de Dias et al. (2), en ejemplares de raza Caracú y Cavalcante et al. (15), en ejemplares de raza Nelore (Tabla 1 ).
Los intervalos entre partos más cortos han sido reportados por Padua et al. (3) y Bergmann et al. (7), en la raza Nelore; Durán y Manrique (9), en la raza Lucerna, Álvarez (11), en la raza Hartón del Valle; Joshi et al. (16), en la raza Sahiwal y Velásquez (13) en la raza Velásquez, variando entre 420 y 374 días (Tabla 1 ).

Los intervalos entre partos superiores al observado en este estudio fueron encontrados por: Bourzat et al. (1), en la raza Kouri; Pereira et al. (4), en la Caracú; Martínez et al. (25); Martínez (10) y Martínez y G onzález (14), en la raza Sanmartinero; Tobón et al. (6), en la raza BO N; Balieiro et al. (8), en la raza G ir; 0 ssa (14), en la raza CCC; G aur et al. (17) y Singh et al. (19) en la raza Deoni. El rango de variación fue de 517 a 446 días (Tabla 1 ).

Tabla 2. Análisis de varianza del intervalo entre partos.

\begin{tabular}{lclll}
\hline Fuentes de variación & Grados de libertad & Cuadrados medios & F & Pr $>$ F \\
\hline Año & 22 & 178673,625 & 9,22 & $<0,0001$ \\
Mes & 3 & 53765,97 & 2,78 & $<0,04$ \\
Sexo & 1 & 47508,89 & 2,45 & 0,1175 \\
Número del parto & 3 & 375400,33 & 19,38 & $<0,0001$ \\
\hline
\end{tabular}

$\mathrm{R}^{2}=0,468$

Efecto del año y/o mes del parto. En cuanto al efecto del mes del parto sobre el intervalo entre partos, el análisis de varianza mostró que esta variable ejerció efecto estadístico significativo $(p<0,0001)$, como causa de variación en los intervalos entre partos (Tabla 2).

El efecto del año del parto es, básicamente, un reflejo de las condiciones favorables del clima, con implicaciones en la disponibilidad de forrajes. Desde el punto de vista del mejoramiento genético, el efecto del año impone que las comparaciones entre las vacas deben ser hechas dentro de un mismo año, eliminando así el efecto de esta variable en el intervalo entre partos. 0 tros autores han observado el efecto del año del parto sobre el intervalo entre partos $(2,3,6,7,15,21,22)$. En contraste, en la misma raza pero en años diferentes Hernández et al. (20), no encontraron efecto significativo del año sobre el intervalo entre partos.
En cuanto al efecto del mes del parto sobre el intervalo entre partos, el análisis de varianza mostró que esta variable ejerció un efecto estadístico significativo $(p<0,04)$ como causa de variación de dicha variable (Tabla 2 ). Los efectos atribuidos al mes del parto son resultantes, principalmente, de las condiciones de alimentación y manejo dadas a los animales. En el presente estudio los intervalos entre partos más cortos correspondieron a los partos ocurridos en los tres primeros meses de cada año $(428,05 ; 424,53$ y 423,82 días) entre los cuales no existió diferencia significativa, pero si en comparación con las que parieron en abril (467,62 días). Parece razonable admitir que las vacas que parieron durante los tres primeros meses de cada año fueron beneficiadas por las condiciones favorables de alimentación a lo largo de sus gestaciones, proporcionales a buenas condiciones físicas por ocasión del parto. Además de este aspecto, las vacas pierden peso en los tres primeros meses de la 
lactancia, recuperándose al inicio de las lluvias, en donde las condiciones de alimentación son más favorables, reduciéndose el período de servicio y consecuentemente el intervalo entre partos.

Las vacas con partos al final del verano e inicio del invierno tuvieron intervalos entre partos más largos posiblemente debido a las condiciones climáticas por las cuales pasaron durante el período del verano. Además, de la pérdida de peso que acompaña el inicio del amamantamiento, lo que ocurre para alargar el período de recuperación de las hembras, con impacto en el período de servicio e intervalo entre partos.

El efecto significativo del mes con relación al intervalo entre partos fue también observado por otros investigadores $(2,3,7,15,20,21)$.

Efecto del orden del parto. El orden de parto fue una causa importante de variación en la duración de los intervalos entre partos en la raza Romosinuano ( $p<0,0001)$, tal como se observa en la tabla 2. Los mayores intervalos entre partos observados fueron para las vacas entre el 10 y 20 parto $(470,38$ días). A partir del tercer parto existió una tendencia de disminución de los intervalos entre partos, alcanzando los valores más bajos para las vacas de 50 más partos, con 400,59 días. Estos resultados coinciden con otros autores $(4,21$ 25) quienes estudiaron las razas Caracú y Sanmartinero, y efecto del orden del parto sobre el intervalo entre partos.

Se recomiendan mayores cuidados especialmente de asistencia y de alimentación, para las vacas primíparas, en el sentido de reducir los intervalos entre partos, una vez que estas presentan una duración superior a 36,78 días con relación a la media estimada del hato.

Efecto del sexo del ternero. Aunque las vacas que parieron terneros machos tuvieron una duración del intervalo entre partos superior en 11,31 días, con relación a las vacas que parieron hembras, esta diferencia no fue estadísticamente significativa (Tabla 2). En contraste, en Brasil la raza Caracú (21), y Nelore (7), el efecto fue altamente significativo, aspecto que fue atribuido a las influencias del manejo, el nivel nutricional antes y después de las gestaciones y a la duración del período de gestación.

Heredabilidad. El estimativo de la heredabilidad encontrada en este trabajo fue de 0,05 $\pm 0,04$, coincidiendo con los descritos en la literatura consultada, los cuales muestran valores cercanos cero $(2,4)$. Esto significa que el intervalo entre partos en el hato Romosinuano del C. I Turipaná, depende en apenas un $5 \%$ de las variaciones entre los genotipos de los animales y el $95 \%$ depende de otras variaciones. El mejoramiento de este carácter depende, básicamente, de mejores condiciones de alimentación y manejo en general, para que las vacas recuperen rapidamente el peso perdido durante los tres primeros meses de lactancia.

En conclusión, los resultados demostraron que el mes de nacimiento y el número de partos en la raza romosinuana son variables importantes que afectan el intervalo entre partos.

\section{REFERENCIAS}

1. Bourzat D, Idriss A, Zeuch, V. La race Kouri. Une population bovine en danger d'absorption. Boletín de información sobre recursos genéticos animales. 1992; 9: 1526.

2. Dias A, De Queiroz S, De Alburquerque L. Efeito endogamia em características reprodutivas de bovinos da raca Caracú. Rev Soc Bras Zoot 1994; 23: 157-164.
3. Padua J, Munari D, Watanabe Y. Avaliacao de efeitos de ambiente e da repetibilidade de características reprodutivas em bovinos da raca Nelore. Rev Soc Bras Zoot 1994; 23: 126-132.

4. Pereira C, Pereira S, Carneiro N. Relacao genética entre características reprodutivas e produtivas de um rebanho bovino da raca Caracú. Estimativas de parámetros 
genéticos. Arq Bras Med Vet Zootec, 1994; 46: 149-160.

5. Martínez C, González F, Huertas H. Parámetros genéticos y productivos del ganado criollo Sanmartinero en el piedemonte llanero. Características reproductivas. 3 er Congreso Iberoamericano de razas autóctonas y criollas. Santafé de Bogotá. 1996; p.490500 .

6. Tobón Y, Franco C, Mejia M, Martínez C. Evaluación de algunos caracteres reproductivos en el ganado Blanco 0 rejinegro ( $B O N)$, C ebú y sus cruces en zona de ladera. 3 er Congreso Iberoamericano de razas autóctonas y criollas. Santafé de Bogotá, Col. 1996; p.497.

7. Bergmann A, G ressler S, Pereira C, Pereira J. Avaliacao de fatores genéticos e de ambiente sobre algumas características reprodutivas de femeas da raca $\mathrm{N}$ elore em regime de estacao de monta restrita. Arq Bras Med Vet Zootec 1998; 50: 633-645.

8. Balieiro S, Pereira J, Verneque R, Pereira C, Bergmann A. Estimativas de parámetros genéticos e de tendencias fenotípica, genéticas e de ambiente de algumas características reprodutivas na raca Gir. Arq Bras Med Vet Zootec 1999; 51: 371376.

9. Duran V, Manrique P. Potencial genético y productivo de la raza bovina de doble propósito Lucerna. Censo y caracterización de los sistemas de producción del ganado criollo y colombiano. Septiembre 23 Santafé de Bogotá. 1999; p.104-107.

10. Martínez G. El ganado criollo Sanmartinero y su potencial productivo. Censo y caracterización de los sistemas de producción del ganado criollo y colombiano. Septiembre 23. Santafé de Bogotá, D.C. Colombia. 1999; 128-138.

11. Álvarez A. Potencial genético y productivo del ganado criollo Hartón del Valle. Censo y caracterización de los sistemas de producción del ganado criollo y colombiano. Septiembre 23. Santafé de Bogotá. 1999; p.94-103.

12. O ssa G. Comportamiento productivo del ganado Costeño con Cuernos y sus cruces. Censo y caracterización de los sistemas de producción del ganado Criollo y colombiano. Septiembre. 23. Santafé de Bogotá. 1999; p.90-93.

13. Velásquez S. Potencial genético y productivo del ganado Velásquez. Censo y caracterización de los sistemas de producción del ganado criollo y colombiano, Santafé de Bogotá, Septiembre 23. 1999; p.139-144.

14. Martínez G, González F. El ganado Sanmartinero (SM) y su potencial productivo. Boletín de Información sobre Recursos $\mathrm{G}$ enéticos Animales. (FAO ) 2000; 28: 7-17.

15. Cavalcante $F$, Martins $R$, Campello $C$, Esteves $M$, Duraes $C$. Intervalo de partos em rebanho Nelore na Amazonia 0 riental. Rev Soc Bras Zootec 2000; 29: 1327 1331.

16. Joshi K, G andhi S. Performance evaluation, conservation and improvement of Sahiwal cattle in India. Boletín de información sobre Recursos $\mathrm{G}$ enéticos Animales (FAO). $2001 ; 31: 43-54$.

17. Gaur K, Kaushit N, Garg C. O ngote cattle in India. Boletín de Información sobre Recursos G enéticos Animales. (FAO). 2002; 32: 27-34.

18. O ssa G, Moreno L, Manrique C, Tobón C, Pérez J, Tarazona G, et al. El mejoramiento genético como instrumento de eficiencia en una empresa de producción bovina. Manual Técnico. Corpoica, Bogotá. 2002; p.42.

19. Singh G, Nivsarkar E, Patil R, Mitkari R. Deoni cattle breed of India. A study on population dynamic and morphometric characteristic. Boletín de Información sobre Recursos $G$ enéticos Animales. (FAO). $2002 ; 32: 36-43$. 
20. Hernández G, Koch M, Dickerson E. Influencia de algunos factores en el intervalo entre partos en ganado Romosinuano. Mem Asoc Latinoam Prod Anim México, 1971; 6:167.

21. Pereira C, Pereira S, Lemos M. Estudo dos fatores a mbientes e genéticos relacionados com o intervalo entre partos na raca Caracú. Arq Esc Vet UFM G, 1980; 32: 81 91.

22. Gómez J, González F, Tobón C, Martínez $G$. Influencias ambientales que afectan el comportamiento productivo del ganado Sanmartinero en la altillanura colombiana. Edad al primer parto e intervalo entre partos. 3er Congreso Iberoamericano de razas autóctonas y criollas. Santafé de Bogotá. 1996; p.511- 512.
23. SAS Institute Inc. SAS/STATTM. SAS user's guide for windows enviroment. 6.08 ed, Cary, SAS Institute Inc, 1995.

24. Boldman G, Kriese A, Van Vleck D, Van Tassell P, Kachaman D. A manual for use of MTDFREML a set of programs to obtain estimates of variance and covariance. (Draft). U.S. Departament o Agricultura, Agricultural Reseach Service. 1995; p.120.

25. Martínez C. Censo y caracterización de los sistemas de producción del ganado criollo y colombiano. Censo y caracterización de los sistemas de producción del ganado criollo y colombiano. Septiembre 23. Santafé de Bogotá, D.C. Colombia. 1999; p.13-64. 